\title{
Orbifold aspects of the Longo-Rehren subfactors
}

\author{
NobuYa SATO* \\ Department of Mathematics, Rikkyo University \\ Nishi-Ikebukuro, Tokyo, 171-8501, JAPAN \\ e-mail: nobuya@rkmath.rikkyo.ac.jp
}

November 13, 2018

\begin{abstract}
In this article, we will prove that the subsectors of $\alpha$-induced sectors for $M \rtimes \hat{G} \supset M$ forms a modular category, where $M \rtimes \hat{G}$ is the crossed product of $M$ by the group dual $\hat{G}$ of a finite group $G$. In fact, we will prove that it is equivalent to Müger's crossed product. By using this identification, we will exhibit an orbifold aspect of the quantum double of $\Delta$ (not necessarily non-degenerate) obtained from a Longo-Rehren inclusion $A \supset B_{\Delta}$ under certain assumptions.

We will apply the above description of the quantum double of $\Delta$ to the Reshetikhin-Turaev topological invariant of closed 3-manifolds, and we obtain a simpler formula, which is a degenerate version of Turaev's theorem that the Reshetikhin-Turaev invariant for the quantum double of a modular category $\hat{\Delta}$ is the product of Reshetikhin-Turaev invariant of $\hat{\Delta}$ and its complex conjugate.
\end{abstract}

\section{Introduction}

Orbifold phenomena have sometimes appeared in subfactor theory. The first appearance was to construct subfactors with Dynkin diagrams of type $D_{2 n}$ out of subfactors with Dynkin diagrams of type $A_{4 n-3}$ through Dynkin diagram automorphisms [15]. This method in consequence suggested the orbifold construction removes the degeneracy of braiding, and it is in fact proved in [10]. Along the same line, in 8] Evans and Kawahigashi extended the method of orbifold construction to the Hecke algebra subfactors of Wenzl 32]. In a more sophisticated way, Goto defined an orbifold subfactor as a simultaneous crossed product by non-strongly outer automorphism with the trivial Loi invariant [11].

*Supported by the Grants-in-Aid for Scientific Research, JSPS. 
There is another known way to remove degeneracy of braiding. That is the quantum double construction. Originally, it is the way to construct a higher symmetric Hopf algebra out of the initial Hopf algebra and its dual Hopf algebra. In subfactor context, we have Ocneanu's asymptotic inclusion $M_{\infty} \supset M \vee M^{o p}$ constructed from a hyperfinite $\mathrm{II}_{1}$ subfactor $N \subset M$. The finite system of $M_{\infty}-M_{\infty}$ bimodules obtained from an asymptotic inclusion is known to form a modular category. In fact, it is the so called center construction in category theory 14. However, this correspondence is not obvious because Ocneanu's construction of the finite system of $M_{\infty}-M_{\infty}$ bimodules makes an ingenious use of topological quantum field theory in three dimensions in the sense of Atiyah [1]. For infinite factors, Longo and Rehren introduced an interesting subfactor nowadays called the Longo-Rehren subfactor. The Longo-Rehren subfactor produces the same tensor category as the one the asymptotic inclusion does [20].

In his paper [13], Izumi examined and clarified the structure of a LongoRehren subfactor and its quantum double in a completely algebraic way, i.e., without using any help of TQFT. Moreover, he proved that the quantum double obtained from a Longo-Rehren inclusion is a modular category and further gave the description of modular $S$ - and $T$-matrices in the language of sectors. Thus, the quantum double in subfactors also provides a machinery to remove degeneracy of braiding. See [10, 21] for the relationship between the orbifold construction and the asymptotic (or Longo-Rehren) inclusion.

In [10, Evans and Kawahigashi proved that the quantum double of a finite system of bimodules with non-degenerate braiding $\hat{\Delta}$ is equivalent to $\hat{\Delta} \otimes \hat{\Delta}^{o p}$ as tensor categories. It often happens that a subsystem $\Delta$ of a finite system of non-degenerate braiding $\hat{\Delta}$ is degenerate. A typical example is $\hat{\Delta}=$ a full system of WZW $S U(N)_{k}$-model and $\Delta=$ the grading 0 part of $\hat{\Delta}$. (The grading is introduced by the cyclic group $\mathbb{Z}_{N}$ acting on the set of integrable highest weight modules of level $k$. [17]) In the case of $S U(2)_{k}$ and $S U(3)_{k}$, they succeeded to describe the quantum double of $\Delta$ in terms of $\hat{\Delta}[10$. Later, by using sector theory, Izumi obtained the quantum double of $\Delta$ in the case of $S U(N)_{k}$ which description is quite close to Müger's crossed product, namely dividing the double category $\hat{\Delta} \otimes \hat{\Delta}^{o p}$ by the group symmetry $\mathbb{Z}_{N}$. In this paper, we will generalize Izumi's argument to obtain the description of the quantum double of $\Delta$ in the language of Müger's crossed product, under the assumption that $\hat{\Delta}$ is a minimal non-degenerate extension i.e., $\hat{\Delta} \cap \Delta^{\prime}=\Delta \cap \Delta^{\prime}$.

Müger's theory of crossed product has its origin at a conjecture by Rehren 27]: Extending endomorphisms on the observable algebra to the ones on the field algebra removes the degeneracy of the braiding. Müger solved this conjecture in 22] and he noticed that it could be possible to formulate the whole theory in terms of tensor category 24. His formulation crucially depends on Doplicher-Roberts duality theory [6, 7]. (See 34] for another equivalent approach to crossed products.) It should be mentioned that at almost the same time as Müger's work, Bruguières developed how to construct a mod- 
ular category out of a certain ribbon category, based on Deligne's internal characterization of tannakien category in characteristic 0 .

These modularizations have obvious applications to the Reshetikhin-Turaev TQFT. Bruguières himself examined some cases such as $S L(N)$ and $P S L(N)$ as examples [5]. Sawin used Müger's machinery to obtain a modular category out of closed subsets of the Weyl alcove of a simple Lie algebra, which is essentially the case dividing some ribbon categories associated with simple Lie algebras by the cyclic group actions. He also obtained a topological invariant of closed 3-manifolds associated with such modular categories [30].

Since we have Longo-Rehren inclusions $A \supset B_{\Delta} \supset B_{\hat{\Delta}}$ for a minimal non-degenerate extension $\hat{\Delta} \supset \Delta$, we can construct the Reshetikhin-Turaev invariant from the data of the quantum double of $\Delta$. As an application of an orbifold aspect of the inclusions $A \supset B_{\Delta} \supset B_{\hat{\Delta}}$, we will have a simpler description of the Reshetikhin-Turaev invariant of closed 3-manifolds constructed from the quantum double of $\Delta$.

This article is organized as follows.

In Sec.2, we collect some terminologies we need in this article. In particular, we will make quick (and somewhat brutal) reviews on the $\alpha$-induction and Müger's theory of crossed product. In Sec.3, we explicitly compute the $\alpha$ induction for the subfactor $M \rtimes \hat{G} \supset M$, where $M \rtimes \hat{G}$ is the crossed product factor by group dual. We will prove that the subsectors of $\alpha$-induced sectors for $M \rtimes \hat{G} \supset M$ forms a modular category. This result is a folklore among the experts, but to the best of author's knowledge, there is no description in the present fashion. In Sec.4, we construct the Longo-Rehren inclusions $A \supset$ $B_{\Delta} \supset B_{\hat{\Delta}}$ from a minimal non-degenerate extension $\hat{\Delta} \supset \Delta$, and we will prove that $B_{\Delta} \supset B_{\hat{\Delta}}$ is conjugate to $B_{\hat{\Delta}} \rtimes \hat{G} \supset B_{\hat{\Delta}}$. This implies that the quantum double of $\Delta$ can be described by $\hat{\Delta}$ and Müger's crossed product. In Sec.5, we will apply the result obtained in Sec.4 to the Reshetikhin-Turaev invariant constructed from the quantum double of $\Delta$. Combined with the result in [16], we can have the statement that the Turaev-Viro-Ocneanu invariant constructed from $\Delta$ is described by the sum of the product of the framed link invariant constructed from $\hat{\Delta}$ and its complex conjugate, which gives a special case of Ocneanu's theorem [26].

Acknowledgment The author would like to thank Professors Y. Kawahigashi and M. Izumi for valuable discussions in the early stage of this work. He also thanks Professor Yamagami and Dr. Müger for comments on the preliminary version of this manuscript.

\section{Preliminaries}

\subsection{Braided system of endomorphisms}

Basics on sector theory. Let $M, N$ be infinite factors, and we denote by $\operatorname{Mor}(N, M)_{0}$ the set of unital normal $*$-homomorphisms from $N$ to $M$ whose 
image has a finite index. The statistical dimension $d(\rho)$ of $\rho \in \operatorname{Mor}(N, M)_{0}$ is given by $d(\rho)=[M: \rho(N)]^{1 / 2} . \quad \rho \in \operatorname{Mor}(N, M)_{0}$ is called irreducible if $M \cap \rho(N)^{\prime} \cong \mathbb{C} 1_{M}$. For $\rho, \sigma \in \operatorname{Mor}(N, M)_{0}$, the intertwiner space $\operatorname{Hom}(\rho, \sigma)$ is defined by $\operatorname{Hom}(\rho, \sigma)=\{V \in M \mid V \rho(x)=\sigma(x) V, x \in N\}$. For every $\rho \in \operatorname{Mor}(N, M)_{0}$, there are isometries $R_{\rho} \in \operatorname{Hom}(i d, \bar{\rho} \rho), \bar{R}_{\rho} \in \operatorname{Hom}(i d, \rho \bar{\rho})$ satisfying $\bar{R}_{\rho}^{*} \rho\left(R_{\rho}\right)=\frac{1}{d(\rho)}, R_{\rho}^{*} \bar{\rho}\left(\bar{R}_{\rho}\right)=\frac{1}{d(\rho)}$. $\bar{\rho}$ is called the conjugate of $\rho$.

The unitary equivalence class of $\rho \in \operatorname{Mor}(N, M)_{0}$ is called a sector, and we denote by $[\rho]$ the sector of $\rho$. For sectors $\left[\rho_{1}\right],\left[\rho_{2}\right],[\rho], \rho_{1}, \rho_{2}, \rho \in \operatorname{Mor}(M, M)_{0}$, we have the product $\left[\rho_{1}\right]\left[\rho_{2}\right]=\left[\rho_{1} \cdot \rho_{2}\right]$, the conjugation $\overline{[\rho]}=[\bar{\rho}]$ and the direct sum: $\left[\rho_{1}\right] \oplus\left[\rho_{2}\right]=[\rho]$, where $\rho(x)=t_{1}^{*} \rho_{1}(x) t_{1}+t_{2}^{*} \rho_{2}(x) t_{2}$ and $t_{1}, t_{2} \in M$ satisfying $t_{i}^{*} t_{j}=\delta_{i, j} 1$ and $t_{1} t_{1}^{*}+t_{2} t_{2}^{*}=1$.

Let $M \supset N$ be an inclusion of infinite factors with finite index $\lambda=[M$ : $N]$ and $\gamma$ be its canonical endomorphism. Then, it is known that there exist isometries $v \in \operatorname{Hom}(i d, \gamma)$ and $\operatorname{Hom}\left(\gamma, \gamma^{2}\right)$ satisfying

$$
v^{*} w=w^{*} \gamma(v)=\lambda^{-1 / 2} 1, w^{*} \gamma(w)=w w^{*}, \gamma(w) w=w^{2} .
$$

Moreover, $M=N v$ and the conditional expectation $E$ from $M$ onto $N$ is given by $E(x)=w^{*} \gamma(x) w$.

Braided system of endomorphisms. Let $M$ be an infinite factor, and $\Delta_{0}$ be a system of irreducible endomorphisms in $\operatorname{End}(M)_{0}=\operatorname{Mor}(M, M)_{0}$. More specifically, $\Delta_{0}$ is the set of irreducible normal $*$-endomorphisms of $M$ closed under the following sector operations:

(i) Different elements in $\Delta_{0}$ are inequivalent.

(ii) $i d_{M} \in \Delta_{0}$.

(iii) For every $\xi \in \Delta_{0}$ there exists $\bar{\xi} \in \Delta_{0}$ such that $\overline{[\xi]}=[\bar{\xi}]$.

(iv) There exists a non-negative integer $N_{\xi \eta}^{\zeta}$ such that $[\xi][\eta]=\oplus_{\zeta \in \Delta_{0}} N_{\xi \eta}^{\zeta}[\zeta]$.

We denote by $\Delta$ the subset of $\operatorname{End}(M)_{0}$ whose element is decomposed into finite direct sums of the elements in $\Delta_{0}$ as sectors.

A system of endomorphisms $\Delta_{0}$ is called braided if for any $\lambda, \mu \in \Delta_{0}$ there exists a unitary intertwiner $\varepsilon(\lambda, \mu) \in \operatorname{Hom}(\lambda \cdot \mu, \mu \cdot \lambda)$ with $\varepsilon(i d, \mu)=$ $\varepsilon(\lambda, i d)=1$ satisfying the following (the Braiding-Fusion equations):

For any $\lambda, \mu, \nu \in \Delta_{0}, t \in \operatorname{Hom}(\lambda, \mu \cdot \nu)$,

$$
\begin{array}{r}
\sigma(t) \varepsilon(\lambda, \sigma)=\varepsilon(\mu, \sigma) \mu(\varepsilon(\nu, \sigma)) t \\
t \varepsilon(\sigma, \lambda)=\mu(\varepsilon(\sigma, \nu)) \varepsilon(\sigma, \mu) \sigma(t) \\
\sigma(t)^{*} \varepsilon(\mu, \sigma) \mu(\varepsilon(\nu, \sigma))=\varepsilon(\lambda, \sigma) t^{*} \\
t^{*} \mu(\varepsilon(\sigma, \nu)) \varepsilon(\sigma, \mu)=\varepsilon(\sigma, \lambda) \rho(t)^{*} .
\end{array}
$$

We call above $\varepsilon$ a braiding on $\Delta_{0}$. For a given braiding $\varepsilon(\lambda, \mu)$ on $\Delta_{0}$, unitary intertwiners $\varepsilon(\mu, \lambda)^{*}$ also satisfies the above conditions of the braiding. We will use the notations $\varepsilon^{+}(\lambda, \mu)=\varepsilon(\lambda, \mu)$ and $\varepsilon^{-}(\lambda, \mu)=\varepsilon(\mu, \lambda)^{*}$ to emphasize the difference. 
A braiding on $\Delta_{0}$ can be extended to a braiding on $\Delta$ in the following way:

$$
\varepsilon(\lambda, \mu)=\sum_{i=1}^{n} \sum_{j=1}^{m} s_{j} \mu_{j}\left(t_{i}\right) \varepsilon\left(\lambda_{i}, \mu_{j}\right) \lambda\left(s_{j}^{*}\right) t_{i}^{*},
$$

where $\lambda(x)=\sum_{i=1}^{n} t_{i} \lambda_{i}(x) t_{i}^{*}$ and $\mu(x)=\sum_{j=1}^{m} s_{j} \mu_{j}(x) s_{j}^{*}$ (i.e., $\lambda=\bigoplus_{i=1}^{n} \lambda_{i}$ and $\left.\mu=\oplus_{j=1}^{m} \mu_{j}\right)$.

Degenerate sectors. A sector $\xi \in \Delta$ is said to be degenerate if $\varepsilon^{+}(\xi, \eta)=$ $\varepsilon^{-}(\xi, \eta)$ for every $\eta \in \Delta_{0} . \Delta$ is said to be non-degenerate if $i d_{M}$ is the only degenerate sector. We denote the set of all of degenerate sectors in $\Delta$ by $\Delta^{d}$ and the set of all of irreducible sectors in $\Delta^{d}$ by $\Delta_{0}^{d}$. Note that $\Delta^{d}$ is a symmetric $C^{*}$-tensor subcategory of $\Delta$ with direct sums, subobjects and conjugates.

For $\xi \in \Delta_{0}^{d}, \phi_{\xi}(\varepsilon(\xi, \xi))=\lambda_{\xi} \in \mathbb{C}$, where $\phi_{\xi}$ is the standard left inverse of $\xi$. The polar decomposition of $\lambda_{\xi}$ is given by $\frac{\omega_{\xi}}{d(\xi)}$. It is easy to show that $\omega_{\xi}= \pm 1$ for $\xi \in \Delta^{d}$ (more generally, for an object in a symmetric $C^{*}$-tensor category). $\Delta^{d}$ is said to be even if $\omega_{\xi}=1$ for every irreducible $\xi \in \Delta^{d}$. We assume $\Delta^{d}$ is even in the sequel. Then, by Doplicher-Roberts duality theory, there exists a finite group $G$ up to isomorphism such that $\Delta^{d} \cong \hat{G}$, where $\hat{G}$ is a category of finite dimensional unitary representations of $G$.

$\alpha$-induction. Let $M \supset N$ be an inclusion of infinite factors with finite index and $\gamma$ be its canonical endomorphism. Let $\Delta_{0} \subset \operatorname{End}(N)_{0}$ be a braided system of endomorphisms with a braiding $\varepsilon$. We define the $\alpha$-induced endomorphism of $\lambda \in \Delta_{0} \alpha_{\lambda} \in \operatorname{End}(M)$ by

$$
\alpha_{\lambda}=\gamma^{-1} \cdot \operatorname{Ad}(\varepsilon(\lambda, \theta)) \cdot \lambda \cdot \gamma
$$

where $\theta=\left.\gamma\right|_{N}$.

The systematic use of $\alpha$-induction was first made by $\mathrm{Xu}$ [33], and further studied in a series of papers by Böckenhauer and Evans [2, 3, 4]. We list some properties of the $\alpha$-induction [2, 33]:

(i) $d\left(\alpha_{\lambda}\right)=d(\lambda)$

(ii) $\alpha_{\lambda} \cdot \alpha_{\mu}=\alpha_{\lambda \cdot \mu}$ for any $\lambda, \mu \in \Delta_{0}$

(iii) $\alpha_{\mu} \cdot \alpha_{\lambda}=\operatorname{Ad}(\varepsilon(\lambda, \mu)) \cdot \alpha_{\lambda} \cdot \alpha_{\mu}$ for any $\lambda, \mu \in \Delta_{0}$

(iv) If $[\lambda]=\left[\lambda_{1}\right] \oplus\left[\lambda_{2}\right], \lambda, \lambda_{1}, \lambda_{2} \in \Delta$, then $\left[\alpha_{\lambda}\right]=\left[\alpha_{\lambda_{1}}\right] \oplus\left[\alpha_{\lambda_{2}}\right]$ and

(v) $\left[\alpha_{\bar{\lambda}}\right]=\left[\overline{\alpha_{\lambda}}\right], \lambda \in \Delta_{0}$.

The $\alpha$-induction on $\Delta_{0}$ is extended to the one on $\Delta$ preserving the above properties.

\subsection{Premodular categories and Müger's crossed prod- uct}

To define Müger's crossed product, we need some terminologies from category theory. See [19] for the basics on $C^{*}$-tensor category and 23] for the full description of crossed product. 
Assumption 2.1. We assume that $\mathcal{C}$ is a $C^{*}$-tensor category with conjugate, direct sums, subobjects, irreducible unit object $\iota$ and a unitary braiding $\varepsilon$.

We use the following notations which are popular in the context of the algebraic quantum field theory:

We use small Greek letters $\rho, \sigma$ etc for objects of $\mathcal{C}$, and the tensor product is denoted by $\rho \sigma$ instead of $\rho \otimes \sigma$. For operations of arrows, we denote the composition of arrows $S \in \operatorname{Hom}(\rho, \sigma), T \in \operatorname{Hom}(\sigma, \tau)$ by $T \circ S \in$ $\operatorname{Hom}(\rho, \tau)$, the tensor product of $S \in \operatorname{Hom}\left(\rho_{1}, \sigma_{1}\right), T \in \operatorname{Hom}\left(\rho_{2}, \sigma_{2}\right)$ by $S \times T \in \operatorname{Hom}\left(\rho_{1} \rho_{2}, \sigma_{1} \sigma_{2}\right)$. We denote by $\mathcal{C}_{0}$ the set of isomorphism classes of irreducible objects.

We remark that under Assumption $2.1 \mathcal{C}$ is a ribbon category and we denote a twist for each irreducible object $\rho \in \mathcal{C}$ by $\omega_{\rho}$.

Since we assume that $\mathcal{C}$ has a conjugate $\bar{\rho}$ for each object $\rho$, there are $R_{\rho} \in \operatorname{Hom}(\iota, \bar{\rho} \rho)$ and $\bar{R}_{\rho} \in \operatorname{Hom}(\iota, \rho \bar{\rho})$ satisfying

$$
\bar{R}_{\rho}^{*} \times i d_{\rho} \circ i d_{\rho} \times R_{\rho}=i d_{\rho}, R_{\rho}^{*} \times i d_{\rho} \circ i d_{\bar{\rho}} \times \bar{R}_{\rho}=i d_{\rho} .
$$

Then, the dimension of an irreducible object $\rho$ is defined by $d(\rho)=R_{\rho}{ }^{*} \circ R_{\rho}$, which takes its value in $[1, \infty)$. (This definition of the dimension extends to reducible objects.)

If the set $\mathcal{C}_{0}$ is finite, the category is called rational. Then, its dimension is defined by $\operatorname{dim} \mathcal{C}=\sum_{\xi \in \mathcal{C}_{0}} d(\xi)^{2}$. In subfactor context, this is called the global index.

When $\mathcal{C}$ is rational, then we set the complex number

$$
S^{\prime}(\xi, \eta) i d_{\iota}=\left(R_{\xi}^{*} \times \bar{R}_{\eta}^{*}\right) \circ\left(i d_{\bar{\xi}} \times \varepsilon(\eta, \xi) \circ \varepsilon(\xi, \eta) \times i d_{\bar{\eta}}\right) \circ\left(R_{\xi} \times \bar{R}_{\eta}\right)
$$

for $\xi, \eta \in \mathcal{C}_{0}$. One can prove that $S^{\prime}(\xi, \eta)$ does not depend on the choice of representatives of $\xi$ and $\eta$.

If $S^{\prime}$ is invertible, $\mathcal{C}$ is called modular. When $\mathcal{C}$ is modular, the matrices

$$
S=\operatorname{dim} \mathcal{C}^{-\frac{1}{2}} S^{\prime}, T=\left(\frac{\Delta_{\mathcal{C}}}{\left|\Delta_{\mathcal{C}}\right|}\right)^{\frac{1}{3}} \operatorname{Diag}\left(\omega_{\xi}\right)
$$

are unitaries and satisfy the relations

$$
S^{2}=(S T)^{3}=C, T C=C T,
$$

where $\Delta_{\mathcal{C}}=\sum_{\xi \in \mathcal{C}_{0}} d(\xi)^{2} \omega(\xi)^{-1}$ and $C=\delta_{\xi, \bar{\eta}}$.

Definition 2.2. If $\mathcal{C}$ satisfies Assumption 2.1 and is rational, we say $\mathcal{C}$ is $C^{*}$-premodular.

For a $C^{*}$-premodular category $\mathcal{C}$ and its full subcategory $\mathcal{S}$, we define $\mathcal{C} \cap \mathcal{S}^{\prime}$, a full subcategory of $\mathcal{C}$, by $\operatorname{Obj} \mathcal{C} \cap \mathcal{S}^{\prime}=\{\rho \in \mathcal{C} \mid \varepsilon(\sigma, \rho) \circ \varepsilon(\rho, \sigma)=$ $i d_{\rho \sigma}$ for all $\left.\sigma \in \mathcal{S}\right\}$. We remark that if $\mathcal{C}$ is modular we have $\operatorname{dim} \mathcal{C} \cap \mathcal{S}^{\prime}=$ $\frac{\operatorname{dim} \mathcal{C}}{\operatorname{dim} \mathcal{S}}$ by Theorem 3.2 [25]. 
Let $\mathcal{C}$ be a $C^{*}$-premodular category and we set $\mathcal{D}_{\mathcal{C}}=\mathcal{C} \cap \mathcal{C}^{\prime}$. We assume that $\mathcal{D}_{\mathcal{C}}$ is even, i.e., twist $\omega_{\xi}=1$ for each irreducible object $\xi$. Then, by Doplicher-Roberts duality theory [6, 7], there is a finite group such that $\mathcal{D}_{\mathcal{C}}$ is equivalent to $U(G)$ as symmetric tensor $*$-categories with conjugates, where $U(G)$ is a category of finite dimensional unitary representations of $G$. In the following, we use the symbol $\otimes$ for the tensor product of $U(G)$.

Let $F$ be an invertible functor from $\mathcal{D}_{\mathcal{C}}$ to $U(G)$ which gives the equivalence, $\hat{G}$ be the set of all isomorphism classes of irreducible objects in $\mathcal{D}_{\mathcal{C}}$, $\left\{\gamma_{k} \mid k \in \hat{G}\right\}$ be a section of objects in $\mathcal{D}_{\mathcal{C}}$ such that $\gamma_{0}=\iota$ and $\mathcal{H}_{k}=F\left(\gamma_{k}\right)$. We choose an orthonormal basis $\left\{V_{k, l}^{m, \alpha}\right\}_{\alpha=1}^{N_{k l}^{m}}$ of $\operatorname{Hom}\left(\gamma_{m}, \gamma_{k} \gamma_{l}\right)$.

Then, a category $\mathcal{C} \rtimes_{0} \mathcal{D}_{\mathcal{C}}$ is defined in the following manner.

- Obj $\mathcal{C} \rtimes_{0} \mathcal{D}_{\mathcal{C}}=\operatorname{Obj} \mathcal{C}$ with the same tensor product as $\mathcal{C}$

- $\operatorname{Hom}_{\mathcal{C} \rtimes_{0} \mathcal{D}_{\mathcal{C}}}(\rho, \sigma)=\bigoplus_{k \in \hat{G}} \operatorname{Hom}_{\mathcal{C}}\left(\gamma_{k} \rho, \sigma\right) \otimes \mathcal{H}_{k}$.

Let $k, l \in \hat{G}, S \otimes \psi_{k} \in \operatorname{Hom}_{\mathcal{C} \rtimes_{0} \mathcal{D}_{\mathcal{C}}}(\rho, \sigma)$ and $T \otimes \psi_{l} \in \operatorname{Hom}_{\mathcal{C} \rtimes_{0} \mathcal{D}_{\mathcal{C}}}(\sigma, \tau)$, where $T \in \operatorname{Hom}\left(\gamma_{l} \rho, \sigma\right), S \in \operatorname{Hom}\left(\gamma_{k} \sigma, \tau\right)$ and $\psi_{k} \in \mathcal{H}_{k}, \psi_{l} \in \mathcal{H}_{l}$. We define the composition of arrows $S \otimes \psi_{k} \circ T \otimes \psi_{l} \in \operatorname{Hom}_{\mathcal{C} \rtimes_{0} \mathcal{D}_{\mathcal{C}}}(\rho, \tau)$ by

$$
S \otimes \psi_{k} \circ T \otimes \psi_{l}=\bigoplus_{k \in \hat{G}} \sum_{\alpha=1}^{N_{k l}^{m}} S \circ i d_{\gamma_{k}} \times T \circ V_{k, l}^{m, \alpha} \times i d_{\rho} \otimes F\left(V_{k, l}^{m, \alpha}\right)^{*}\left(\psi_{k} \otimes \psi_{l}\right)
$$

and extend this linearly.

Let $k, l \in \hat{G}, S \otimes \psi_{k} \in \operatorname{Hom}_{\mathcal{C} \rtimes_{0} \mathcal{D}_{\mathcal{C}}}\left(\rho_{1}, \sigma_{1}\right)$ and $T \otimes \psi_{l} \in \operatorname{Hom}_{\mathcal{C} \rtimes_{0} \mathcal{D}_{\mathcal{C}}}\left(\rho_{2}, \sigma_{2}\right)$, where $S \in \operatorname{Hom}\left(\gamma_{k} \rho_{1}, \sigma_{1}\right), T \in \operatorname{Hom}\left(\gamma_{l} \rho_{2}, \sigma_{2}\right)$ and $\psi_{k} \in \mathcal{H}_{k}, \psi_{l} \in \mathcal{H}_{l}$. We define the tensor product of arrows $S \otimes \psi_{k} \times T \otimes \psi_{l} \in \operatorname{Hom}_{\mathcal{C} \rtimes_{0} \mathcal{D}_{\mathcal{C}}}\left(\rho_{1} \rho_{2}, \sigma_{1} \sigma_{2}\right)$ by

$$
\begin{gathered}
S \otimes \psi_{k} \times T \otimes \psi_{l}=\bigoplus_{\substack{k \in \hat{G} \\
\alpha=1}} \sum_{k l}^{N_{k l}^{m}} S \times T \circ i d_{\gamma_{k}} \times \varepsilon\left(\gamma_{l}, \rho_{1}\right) \times i d_{\rho_{2}} \circ V_{k, l}^{m, \alpha} \times i d_{\rho_{1} \rho_{2}} \\
\otimes F\left(V_{k, l}^{m, \alpha}\right)^{*}\left(\psi_{k} \otimes \psi_{l}\right)
\end{gathered}
$$

and extend this linearly.

Let $S \otimes \psi \in \operatorname{Hom}_{\mathcal{C} \rtimes_{0} \mathcal{D}_{\mathcal{C}}}(\rho, \sigma)$, where $S \in \operatorname{Hom}\left(\gamma_{k} \rho, \sigma\right)$ and $\psi_{k} \in \mathcal{H}_{k}$. We define the $*$-operation of the arrows $(S \otimes \psi)^{*} \in \operatorname{Hom}_{\mathcal{C} \rtimes_{0} \mathcal{D}_{\mathcal{C}}}(\sigma, \rho)$ by

$$
(S \otimes \psi)^{*}=R_{k}^{*} \times i d_{\rho} \circ i d_{\overline{\gamma_{k}}} \times S^{*} \otimes\left\langle\psi_{k} \otimes \cdot, F\left(\bar{R}_{k}\right) \Omega\right\rangle,
$$

where $\Omega$ is a unit vector in the trivial representation $\mathcal{H}_{0} \cong \mathbb{C}$ such that $\Omega \otimes \psi=\psi \otimes \Omega=\psi$ for all $\psi \in \operatorname{Obj} U(G)$.

It turns out that $\mathcal{C} \rtimes_{0} \mathcal{D}_{\mathcal{C}}$ is a $C^{*}$-tensor category with conjugates and direct sums.

Remark 2.3. For $\mathcal{C}$, we have another braiding $\varepsilon^{-}(\lambda, \mu)=\varepsilon(\mu, \lambda)^{*}$. When we need to clarify which braiding we used, we will write $\mathcal{C} \rtimes_{0,+} \mathcal{D}_{\mathcal{C}}$ and $\mathcal{C} \rtimes_{0,-} \mathcal{D}_{\mathcal{C}}$ depending on the choice of the braiding $\varepsilon^{+}$and $\varepsilon^{-}$, respectively. 
$\mathcal{C} \rtimes_{0} \mathcal{D}_{\mathcal{C}}$ is not closed under subobjects in general. However, we can enlarge $\mathcal{C} \rtimes_{0} \mathcal{D}_{\mathcal{C}}$ to be closed under the subobjects. Such a procedure is called closure in Definition 3.11 in 24]. We denote the closure of $\mathcal{C} \rtimes_{0} \mathcal{D}_{\mathcal{C}}$ by $\mathcal{C} \rtimes \mathcal{D}_{\mathcal{C}}$ and call it the crossed product of $\mathcal{C}$ by $\mathcal{D}_{\mathcal{C}}$. We remark that $\operatorname{dim} \mathcal{C} \rtimes \mathcal{D}_{\mathcal{C}}=\frac{\operatorname{dim} \mathcal{C}}{\operatorname{dim} \mathcal{D}_{\mathcal{C}}}$.

It is important to mention that $\mathcal{C} \rtimes \mathcal{D}_{\mathcal{C}}$ is a modular category due to Theorem 4.4 in [24].

Remark 2.4. Müger has constructed a $C^{*}$-tensor category $\mathcal{C} \rtimes \mathcal{S}$ with conjugates, direct sums and subobjects from $\mathcal{C}$ (not rational in general) and $\mathcal{S}$, where $\mathcal{S}$ is a symmetric $C^{*}$-tensor subcategory of $\mathcal{C}$, not necessarily $\mathcal{S} \subset \mathcal{D}_{\mathcal{C}}$. See 24] for details.

\section{Müger's crossed product and $\alpha$-induction for subfactors}

Let $M, \Delta$ and $\Delta^{d}$ be as in Subsection 2.1, and we assume that $\Delta_{0}$ is a finite set. We further assume that $\Delta^{d}$ is even and $\Delta^{d} \cong U(G)$, where $G$ is a finite group. Then, by Doplicher-Roberts duality theory there exists a factor, denoted by $M \rtimes \hat{G}$, which contains $M$ as a subfactor with index $|G|$. See [ 6 ] and [7] for the detailed accounts.

We may assume that $M \rtimes \hat{G}$ is generated by $M$ and isometries $\left\{\psi_{i}^{(\sigma)}\right.$, $\left.i=1, \cdots, d(\sigma), \sigma \in \Delta_{0}^{d}\right\}$ satisfying :

$$
\begin{gathered}
\psi^{(\iota)}:=\psi_{1}^{(\iota)}=1 \\
\psi_{i}^{(\sigma)^{*}} \psi_{j}^{\left(\sigma^{\prime}\right)}=\delta_{i, j} \delta_{\sigma, \sigma^{\prime}} \\
\sum_{i=1}^{d(\sigma)} \psi_{i}^{(\sigma)} \psi_{i}^{(\sigma)^{*}}=1 \\
\psi_{i}^{(\sigma)} x=\sigma(x) \psi_{i}^{(\sigma)}, x \in M \\
\psi_{i}^{(\rho)} \psi_{j}^{(\sigma)}=\sum_{\tau \in \Delta_{0}^{d}} \sum_{k=1}^{d(\tau)} V_{(\rho, i)(\sigma, j)}^{(\tau, k)} \psi_{k}^{(\tau)} \\
\psi_{i}^{(\sigma)^{*}}=R_{\sigma}^{*} \psi_{i}^{(\bar{\sigma})} \\
\sum_{i=1}^{d\left(\sigma_{1}\right)} \sum_{j=1}^{d\left(\sigma_{2}\right)} \psi_{j}^{\left(\sigma_{2}\right)} \psi_{i}^{\left(\sigma_{1}\right)} \psi_{j}^{\left(\sigma_{2}\right)^{*}} \psi_{i}^{\left(\sigma_{1}\right)^{*}}=\varepsilon\left(\sigma_{1}, \sigma_{2}\right),
\end{gathered}
$$

where $V_{(\rho, i)(\sigma, j)}^{(\tau, k)} \in \operatorname{Hom}(\tau, \rho \cdot \sigma)$ and $R_{\sigma} \in \operatorname{Hom}(\iota, \bar{\sigma} \cdot \sigma)$.

Remark 3.1. (1) It is known that $\left\{\psi_{i}^{(\sigma)}, i=1, \cdots, d(\sigma), \sigma \in \Delta_{0}^{d}\right\}$ is a left $M$-module basis.

(2) When $x=\sum_{\sigma, i} t_{i}^{(\sigma)} \psi_{i}^{(\sigma)} \in M \rtimes \hat{G}$, the conditional expectation $E: M \rtimes$ $\hat{G} \longrightarrow M$ is given by $E(x)=t^{(\iota)}$. By computations, one has $E\left(\psi_{i}^{(\sigma)} \psi_{j}^{(\rho)^{*}}\right)=$ $\delta_{\sigma, \rho} \delta_{i, j} \frac{1}{d(\sigma)}$, where $\lambda=[M \rtimes \hat{G}: M]$.

Lemma 3.2. Let $v=\sum_{\sigma, i} t_{i}^{(\sigma)} \psi_{i}^{(\sigma)} \in \operatorname{Hom}(i d, \gamma)$. Then, we have the relations $t_{i}^{(\sigma)}=d(\sigma) E\left(v \psi_{i}^{(\sigma)^{*}}\right) \in \operatorname{Hom}(\sigma, \theta)$ and $\psi_{i}^{(\sigma)}=\frac{\lambda}{d(\sigma)} t_{i}^{(\sigma)^{*}} v$. Furthermore, $t_{i}^{(\sigma)}, i=1, \cdots, d(\sigma)$ satisfy $t_{i}^{(\sigma)^{*}} t_{j}^{(\rho)}=\delta_{\sigma, \rho} \delta_{i, j} \frac{d(\sigma)}{\lambda}$ and $\sum_{\sigma, i} \frac{\lambda}{d(\sigma)} t_{i}^{(\sigma)} t_{i}^{(\sigma)^{*}}=1$. 
Proof. Applying the conditional expectation $E$ to the equation $v \psi_{j}^{(\rho)^{*}}=$ $\sum_{\sigma, i} t_{i}^{(\sigma)} \psi_{i}^{(\sigma)} \psi_{j}^{(\rho)^{*}}$, we have

$$
E\left(v \psi_{j}^{(\rho)^{*}}\right)=\sum_{\sigma, i} t_{i}^{(\sigma)} E\left(\psi_{i}^{(\sigma)} \psi_{j}^{(\rho)^{*}}\right)=t_{j}^{(\rho)} \frac{1}{d(\rho)} .
$$

Therefore, $t_{i}^{(\sigma)}=d(\sigma) E\left(v \psi_{i}^{(\sigma)^{*}}\right)$. Multiplying $v$ from the left of the equality $\psi_{i}^{(\sigma)^{*}} \sigma(x)=x \psi_{i}^{(\sigma)^{*}}, x \in M$, we have

$$
v \psi_{i}^{(\sigma)^{*}} \sigma(x)=v x \psi_{i}^{(\sigma)^{*}}=\gamma(x) v \psi_{i}^{(\sigma)^{*}}=\theta(x) v \psi_{i}^{(\sigma)^{*}} .
$$

Apply the conditional expectation $E$ to the above equality, then we have

$$
E\left(v \psi_{i}^{(\sigma)^{*}}\right) \sigma(x)=\theta(x) E\left(v \psi_{i}^{(\sigma)^{*}}\right) .
$$

Hence, $t_{i}^{(\sigma)}=d(\sigma) E\left(v \psi_{i}^{(\sigma)^{*}}\right) \in \operatorname{Hom}(\sigma, \theta)$.

Let us compute $t_{i}^{(\sigma)^{*}} t_{j}^{(\rho)}$.

$$
\begin{aligned}
{t_{i}^{(\sigma)^{*}} t_{j}^{(\rho)}}=d(\sigma) d(\rho) E\left(\psi_{i}^{(\sigma)} v^{*}\right) E\left(v \psi_{j}^{(\rho)^{*}}\right) \\
=d(\sigma) d(\rho) w^{*} \gamma\left(\psi_{i}^{(\sigma)} v^{*}\right) w w^{*} \gamma\left(v \psi_{j}^{(\rho)^{*}}\right) w \\
=d(\sigma) d(\rho) w^{*} \gamma\left(\psi_{i}^{(\sigma)}\right) \gamma\left(v^{*}\right) w w^{*} \gamma(v) \gamma\left(\psi_{j}^{(\rho)^{*}}\right) w \\
=\lambda^{-1} d(\sigma) d(\rho) w^{*} \gamma\left(\psi_{i}^{(\sigma)} \psi_{j}^{(\rho)^{*}}\right) w \\
=\lambda^{-1} d(\sigma) d(\rho) E\left(\psi_{i}^{(\sigma)} \psi_{j}^{(\rho)^{*}}\right) \\
=\delta_{\sigma, \rho} \delta_{i, j} \frac{d(\sigma)}{\lambda} .
\end{aligned}
$$

Next, we compute $t_{i}^{(\sigma)^{*}} v$.

$$
\begin{aligned}
t_{i}^{(\sigma)^{*}} v & =d(\sigma) E\left(\psi_{i}^{(\sigma)} v^{*}\right) v \\
& =d(\sigma) w^{*} \gamma\left(\psi_{i}^{\sigma}\right) \gamma\left(v^{*}\right) w v \\
& =\lambda^{-1 / 2} d(\sigma) w^{*} \gamma\left(\psi_{i}^{(\sigma)}\right) v \\
& =\lambda^{-1 / 2} d(\sigma) w^{*} v \psi_{i}^{(\sigma)} \\
& =\frac{d(\sigma)}{\lambda} \psi_{i}^{(\sigma)} .
\end{aligned}
$$

Hence, $\psi_{i}^{(\sigma)}=\frac{\lambda}{d(\sigma)} t_{i}^{(\sigma)^{*}} v$.

Then, we have the following identity

$$
v=\sum_{\sigma, i} t_{i}^{(\sigma)} \psi_{i}^{(\sigma)}=\sum_{\sigma, i} \frac{\lambda}{d(\sigma)} t_{i}^{(\sigma)} t_{i}^{(\sigma)^{*}} v .
$$

Multiplying $v^{*}$ from the right of the above equality and applying $E$, then we have

$$
\sum_{\sigma, i} \frac{\lambda}{d(\sigma)} t_{i}^{(\sigma)} t_{i}^{(\sigma)^{*}}=1
$$

This completes the proof. 
Proposition 3.3. The equation (3.15) is equivalent to the identity $\varepsilon(\theta, \theta) v^{2}=$ $v^{2}$.

Proof. By Lemma $3.2, \sqrt{\frac{\lambda}{d(\sigma)}} t_{i}^{(\sigma)}$ is isometry in $\operatorname{Hom}(\sigma, \theta)$. Hence, $\varepsilon(\theta, \theta)$ is given by

$$
\begin{aligned}
\varepsilon(\theta, \theta) & =\sum_{\sigma, \sigma^{\prime}} \sum_{i, j} \sqrt{\frac{\lambda}{d\left(\sigma^{\prime}\right)}} t_{j}^{\left(\sigma^{\prime}\right)} \sigma^{\prime}\left(\sqrt{\frac{\lambda}{d(\sigma)}} t_{i}^{(\sigma)}\right) \varepsilon\left(\sigma, \sigma^{\prime}\right) \sigma\left(\sqrt{\frac{\lambda}{d\left(\sigma^{\prime}\right)}} t_{j}^{\left(\sigma^{\prime}\right)^{*}}\right) \sqrt{\frac{\lambda}{d(\sigma)}} t_{i}^{(\sigma)^{*}} \\
& =\sum_{\sigma, \sigma^{\prime}} \sum_{i, j} \lambda^{2} d(\sigma)^{-1} d\left(\sigma^{\prime}\right)^{-1} t_{j}^{\left(\sigma^{\prime}\right)} \sigma^{\prime}\left(t_{i}^{(\sigma)}\right) \varepsilon\left(\sigma, \sigma^{\prime}\right) \sigma\left(t_{j}^{\left(\sigma^{\prime}\right)^{*}}\right) t_{i}^{(\sigma)^{*}} .
\end{aligned}
$$

Then, we have

$$
\begin{aligned}
\varepsilon(\theta, \theta) v^{2} & =\sum_{\sigma, \sigma^{\prime}, \tau} \sum_{i, j, k} \lambda d\left(\sigma^{\prime}\right)^{-1} t_{j}^{\left(\sigma^{\prime}\right)} \sigma^{\prime}\left(t_{i}^{(\sigma)}\right) \varepsilon\left(\sigma, \sigma^{\prime}\right) \sigma\left(t_{j}^{\left(\sigma^{\prime}\right)^{*}}\right) \psi_{i}^{(\sigma)} t_{k}^{(\tau)} \psi_{k}^{(\tau)} \\
& =\sum_{\sigma, \sigma^{\prime}, \tau} \sum_{i, j, k} \lambda d\left(\sigma^{\prime}\right)^{-1} t_{j}^{\left(\sigma^{\prime}\right)} \sigma^{\prime}\left(t_{i}^{(\sigma)}\right) \varepsilon\left(\sigma, \sigma^{\prime}\right) \sigma\left(t_{j}^{\left(\sigma^{\prime}\right)^{*}} t_{k}^{(\tau)}\right) \psi_{i}^{(\sigma)} \psi_{k}^{(\tau)} \\
& =\sum_{\sigma, \sigma^{\prime}} \sum_{i, j} t_{j}^{\left(\sigma^{\prime}\right)} \sigma^{\prime}\left(t_{i}^{(\sigma)}\right) \varepsilon\left(\sigma, \sigma^{\prime}\right) \psi_{i}^{(\sigma)} \psi_{j}^{\left(\sigma^{\prime}\right)}
\end{aligned}
$$

The equation (3.15) is equivalent to $\varepsilon\left(\sigma, \sigma^{\prime}\right) \psi_{i}^{(\sigma)} \psi_{j}^{\left(\sigma^{\prime}\right)}=\psi_{j}^{\left(\sigma^{\prime}\right)} \psi_{i}^{(\sigma)}$, and with this, the formula (3.16) is equal to

$$
\sum_{\sigma, \sigma^{\prime}} \sum_{i, j} t_{j}^{\left(\sigma^{\prime}\right)} \sigma^{\prime}\left(t_{i}^{(\sigma)}\right) \psi_{j}^{\left(\sigma^{\prime}\right)} \psi_{i}^{(\sigma)}=\sum_{\sigma, \sigma^{\prime}} \sum_{i, j} t_{j}^{\left(\sigma^{\prime}\right)} \psi_{j}^{\left(\sigma^{\prime}\right)} t_{i}^{(\sigma)} \psi_{i}^{(\sigma)}=v^{2}
$$

On the contrary, assume that $\varepsilon(\theta, \theta) v^{2}=v^{2}$. Multiplying $\sigma^{\prime}\left(t_{i}^{(\sigma)}\right)^{*} t_{j}^{\left(\sigma^{\prime}\right)^{*}}$ from the left of $\varepsilon(\theta, \theta) v^{2}=\sum_{\sigma, \sigma^{\prime}} \sum_{i, j} t_{j}^{\left(\sigma^{\prime}\right)} \sigma^{\prime}\left(t_{i}^{(\sigma)}\right) \varepsilon\left(\sigma, \sigma^{\prime}\right) \psi_{i}^{(\sigma)} \psi_{j}^{\left(\sigma^{\prime}\right)}$, we have

$$
\sigma^{\prime}\left(t_{i}^{(\sigma)}\right)^{*} t_{j}^{\left(\sigma^{\prime}\right)^{*}} \varepsilon(\theta, \theta) v^{2}=\frac{d\left(\sigma^{\prime}\right) d(\sigma)}{\lambda^{2}} \varepsilon\left(\sigma, \sigma^{\prime}\right) \psi_{i}^{(\sigma)} \psi_{j}^{\left(\sigma^{\prime}\right)}
$$

On the other hand, multiplying $\sigma^{\prime}\left(t_{i}^{(\sigma)}\right)^{*} t_{j}^{\left(\sigma^{\prime}\right)^{*}}$ from the left of $v^{2}$, we have

$$
\sigma^{\prime}\left(t_{i}^{(\sigma)}\right)^{*} t_{j}^{\left(\sigma^{\prime}\right)^{*}} v^{2}=\frac{d\left(\sigma^{\prime}\right) d(\sigma)}{\lambda^{2}} \psi_{j}^{\left(\sigma^{\prime}\right)} \psi_{i}^{(\sigma)}
$$

Thus, $\varepsilon\left(\sigma, \sigma^{\prime}\right) \psi_{i}^{(\sigma)} \psi_{j}^{\left(\sigma^{\prime}\right)}=\psi_{j}^{\left(\sigma^{\prime}\right)} \psi_{i}^{(\sigma)}$.

Remark 3.4. The identity $\varepsilon(\theta, \theta) v^{2}=v^{2}$ is called the chiral locality condition in [4].

Lemma 3.5. For $\lambda \in \Delta$, we have

$$
\alpha_{\lambda}^{ \pm}\left(\psi_{i}^{(\sigma)}\right)=\varepsilon^{ \pm}(\lambda, \sigma)^{*} \psi_{i}^{(\sigma)}
$$

where $\sigma \in \Delta_{0}^{d}, i=1, \cdots, d(\sigma)$. In particular, $\alpha_{\lambda}^{+}=\alpha_{\lambda}^{-}$for $\lambda \in \Delta \cap \Delta^{d^{\prime}}=$ $\left\{\rho_{\xi} \in \Delta \mid \varepsilon(\xi, \sigma) \varepsilon(\sigma, \xi)=1, \forall \sigma \in \Delta_{0}^{d}\right\}$. 
Proof. Let $\gamma$ be the canonical endomorphism of $M \rtimes \hat{G} \supset M$ and $\theta$ the restriction of $\gamma$ to $M$.

Applying $\gamma$ to (3.12), we have $\gamma\left(\psi_{i}^{(\sigma)}\right) \theta(x)=\theta \cdot \sigma(x) \gamma\left(\psi_{i}^{(\sigma)}\right)$. Thus, $\gamma\left(\psi_{i}^{(\sigma)^{*}}\right) \in \operatorname{Hom}(\theta \cdot \sigma, \theta)$. By the Braiding-Fusion equation (2.5),

$$
\varepsilon^{ \pm}(\lambda, \theta)^{*} \theta\left(\varepsilon^{ \pm}(\lambda, \sigma)^{*}\right) \gamma\left(\psi_{i}^{(\sigma)}\right)=\lambda\left(\gamma\left(\psi_{i}^{(\sigma)}\right)\right) \varepsilon^{ \pm}(\lambda, \theta)^{*} .
$$

Applying $\gamma^{-1}$

$$
\varepsilon^{ \pm}(\lambda, \sigma)^{*} \psi_{i}^{(\sigma)}=\gamma^{-1} \cdot A d\left(\varepsilon^{ \pm}(\lambda, \theta)\right) \lambda \cdot \gamma\left(\psi_{i}^{(\sigma)}\right)=\alpha_{\lambda}^{ \pm}\left(\psi_{i}^{(\sigma)}\right) .
$$

The last claim is clear because $\varepsilon^{+}(\lambda, \sigma)=\varepsilon^{-}(\lambda, \sigma)$ for $\lambda \in \Delta \cap \Delta^{d^{\prime}}$.

Lemma 3.6. For $\lambda, \mu \in \Delta$,

$\operatorname{Hom}\left(\alpha_{\lambda}, \alpha_{\mu}\right)=\left\{\sum_{\sigma \in \Delta_{0}^{d}} \sum_{i=1}^{d(\sigma)} t_{i}^{(\sigma)} \psi_{i}^{(\sigma)} ; t_{i}^{(\sigma)} \in \operatorname{Hom}(\sigma \cdot \lambda, \mu), i=1, \cdots, d(\sigma), \sigma \in \Delta_{0}^{d}\right\}$.

Proof. Let $t \in \operatorname{Hom}\left(\alpha_{\lambda}, \alpha_{\mu}\right)$. We may write $t=\sum_{\sigma \in \Delta_{0}^{d}} \sum_{i=1}^{d(\sigma)} t_{i}^{(\sigma)} \psi_{i}^{(\sigma)}$. We remark that this expression is unique. For $x \in M$, we have

$$
\sum_{\sigma, i} t_{i}^{(\sigma)} \psi_{i}^{(\sigma)} \lambda(x)=\sum_{\sigma, i} \mu(x) t_{i}^{(\sigma)} \psi_{i}^{(\sigma)}
$$

Since $\psi_{i}^{(\sigma)} \in \operatorname{Hom}(i d, \sigma)$, the above equality is

$$
\sum_{\sigma, i} t_{i}^{(\sigma)} \sigma \cdot \lambda(x) \psi_{i}^{(\sigma)}=\sum_{\sigma, i} \mu(x) t_{i}^{(\sigma)} \psi_{i}^{(\sigma)}
$$

Thus, $t_{i}^{(\sigma)} \sigma \cdot \lambda(x)=\mu(x) t_{i}^{(\sigma)}$ for any $x \in M, i=1, \cdots, d(\sigma)$ and $\sigma \in \Delta_{0}^{d}$.

For $t$ above, let us show $t \alpha_{\lambda}\left(\psi^{\left(\sigma^{\prime}\right)}\right)=\alpha_{\mu}\left(\psi^{\left(\sigma^{\prime}\right)}\right) t$, where $\psi^{\left(\sigma^{\prime}\right)}$ is an isometry in $\left\{\psi_{i}^{(\sigma)}, i=1, \cdots, d(\sigma), \sigma \in \Delta_{0}^{d}\right\}$. We will show that the left hand side is equal to the right hand side.

$$
\begin{aligned}
\sum_{\sigma, i} t_{i}^{(\sigma)} \psi_{i}^{(\sigma)} \varepsilon\left(\lambda, \sigma^{\prime}\right)^{*} \psi^{\left(\sigma^{\prime}\right)} & =\sum_{\sigma, i} t_{i}^{(\sigma)} \sigma\left(\varepsilon\left(\lambda, \sigma^{\prime}\right)^{*}\right) \psi_{i}^{(\sigma)} \psi^{\left(\sigma^{\prime}\right)} \\
& =\sum_{\sigma, i} \varepsilon\left(\mu, \sigma^{\prime}\right)^{*} \sigma^{\prime}\left(t_{i}^{(\sigma)}\right) \varepsilon\left(\sigma, \sigma^{\prime}\right) \psi_{i}^{(\sigma)} \psi^{\left(\sigma^{\prime}\right)} \\
& =\sum_{\sigma, i} \varepsilon\left(\mu, \sigma^{\prime}\right)^{*} \sigma^{\prime}\left(t_{i}^{(\sigma)}\right) \psi^{\left(\sigma^{\prime}\right)} \psi_{i}^{(\sigma)} \\
& =\sum_{\sigma, i} \varepsilon\left(\mu, \sigma^{\prime}\right)^{*} \psi^{\left(\sigma^{\prime}\right)} t_{i}^{(\sigma)} \psi_{i}^{(\sigma)}
\end{aligned}
$$

where we used the Braiding-Fusion equation (2.2) for the second equality. This completes the proof. 
Remark 3.7. By the above lemma, we have

$$
\operatorname{Hom}\left(i d, \alpha_{\rho}\right)=\left\{\sum_{i=1}^{d(\rho)} t_{i}^{(\rho)} \psi_{i}^{(\rho)} ; t_{i}^{(\rho)} \rho(x)=\rho(x) t_{i}^{(\rho)}, \forall x \in M, i=1, \cdots, d(\rho)\right\}
$$

for $\rho \in \Delta_{0}^{d}$, which is a Hilbert space with dimension $d(\rho)$. Since $d\left(\alpha_{\rho}\right)=d(\rho)$, we conclude that $\alpha_{\rho} \cong \oplus_{i=1}^{d(\rho)} i d$. This can be read that $\alpha$-induction trivializes degenerate sectors.

Let $\lambda \in \Delta \cap \Delta^{d^{\prime}}$ and we use the notation $\alpha_{\lambda}$ instead of $\alpha_{\lambda}^{+}=\alpha_{\lambda}^{-}$. We denote by $\left(\Delta \cap \Delta^{d^{\prime}}\right)^{\alpha}$ the subset of $\operatorname{End}(M \rtimes \hat{G})_{0}$ consisting of subsectors of $\alpha_{\lambda}$, when $\lambda$ varies in $\Delta \cap \Delta^{d^{\prime}}$.

Thanks to Proposition 3.3, we can make a full use of the arguments in the subsection 3.3 in [4]. For this, let $\beta, \delta$ be subsectors of $\alpha_{\lambda}$ and $\alpha_{\mu}$ for some $\lambda, \mu \in \Delta \cap \Delta^{d^{\prime}}$, respectively. We set

$$
\varepsilon_{r}(\beta, \delta)=s^{*} \alpha_{\mu}\left(t^{*}\right) \varepsilon(\lambda, \mu) \alpha_{\lambda}(s) t \in \operatorname{Hom}(\beta \cdot \delta, \delta \cdot \beta)
$$

with isometries $t \in \operatorname{Hom}\left(\beta, \alpha_{\lambda}\right), s \in \operatorname{Hom}\left(\delta, \alpha_{\mu}\right)$. It is proved in Lemma 3.11 [4] that $\varepsilon_{r}(\beta, \delta)$ does not depend on $\lambda, \mu$ and on the isometries $s, t$. Moreover, $\varepsilon_{r}(\beta, \delta)$ for $\beta, \delta \in\left(\Delta \cap \Delta^{d^{\prime}}\right)^{\alpha}$ defines a braiding (called a relative braiding) on $\left(\Delta \cap \Delta^{d^{\prime}}\right)^{\alpha}$ (Corollary $3.13[4]$ ).

Under these preliminaries, we have the following

Proposition 3.8. $\left(\Delta \cap \Delta^{d^{\prime}}\right)^{\alpha}$ is a modular category.

Proof. Let $\alpha_{\lambda}=\oplus_{i=1}^{p} \beta_{i}, \lambda \in \Delta \cap \Delta^{d^{\prime}}$, and $\delta_{j} \in\left(\Delta \cap \Delta^{d^{\prime}}\right)^{\alpha}$ such that $\alpha_{\mu}=\oplus_{j=1}^{q} \delta_{j}$ for some $\mu \in\left(\Delta \cap \Delta^{d^{\prime}}\right)^{\alpha}$. Assume $\varepsilon_{r}\left(\beta_{i}, \delta_{j}\right) \varepsilon_{r}\left(\delta_{j}, \beta_{i}\right)=1$ for all $j=1, \cdots, q$. Then, we have $\varepsilon(\lambda, \delta) \varepsilon(\delta, \lambda)=1$ by Lemma 3.14 [4]. Hence, for $\forall \delta \in \Delta \cap \Delta^{d^{\prime}}$, we have $\varepsilon(\lambda, \delta) \varepsilon(\delta, \lambda)=1$, which implies $\lambda \in \Delta^{d}$.

Since $\alpha_{\lambda}=\oplus_{i=1}^{d(\lambda)} i d$ by Remark [3.7, we have $\beta_{i}=i d$ for all $i=1, \cdots, p$, which proves that $\varepsilon_{r}$ is a non-degenerate braiding on $\left(\Delta \cap \Delta^{d^{\prime}}\right)^{\alpha}$. Thus, $\left(\Delta \cap \Delta^{d^{\prime}}\right)^{\alpha}$ is modular.

So far, we have discussed the similarities to Müger's theory of crossed product. In fact, we have the following

Proposition 3.9. For the inclusion $M \rtimes \hat{G} \supset M$, the image of $\Delta$ by the $\alpha^{ \pm}$-induction is given by $\Delta \rtimes_{0, \pm} \Delta^{d}$. In particular, $\left(\Delta \cap \Delta^{d^{\prime}}\right)^{\alpha}$ is naturally identified with $\left(\Delta \cap \Delta^{d^{\prime}}\right) \rtimes \Delta^{d}$.

Proof. For the composition of the intertwiners, let $s=\sum_{\sigma \in \Delta_{0}^{d}} \sum_{i=1}^{d(\sigma)} s_{i}^{(\sigma)} \psi_{i}^{(\sigma)} \in$ $\operatorname{Hom}\left(\alpha_{\lambda}, \alpha_{\mu}\right), t=\sum_{\rho \in \Delta_{0}^{d}} \sum_{i=1}^{d(\rho)} t_{i}^{(\rho)} \psi_{i}^{(\rho)} \in \operatorname{Hom}\left(\alpha_{\mu}, \alpha_{\nu}\right)$. Then, ts $\in \operatorname{Hom}\left(\alpha_{\lambda}, \alpha_{\nu}\right)$ defines the composition of morphisms $t$ and $s$.

We use the notations $\rho=\gamma_{k}, \sigma=\gamma_{l}, s^{(l)}=s^{\left(\gamma_{l}\right)}, t^{(k)}=t^{\left(\gamma_{k}\right)}, \psi^{(l)}=\psi^{\left(\gamma_{l}\right)}$ and $\psi^{(k)}=\psi^{\left(\gamma_{k}\right)}$, for simplicity. 
It is enough to check the condition for $t=t^{(k)} \psi^{(k)}$ and $s=s^{(l)} \psi^{(l)}$ because of linearity.

$$
\begin{aligned}
t^{(k)} \psi^{(k)} s^{(l)} \psi^{(l)} & =t^{(k)} \gamma_{k}\left(s^{(l)}\right) \psi^{(k)} \psi^{(l)} \\
& =\sum_{\gamma_{m} \in \Delta_{0}^{d}} \sum_{\alpha=1}^{d\left(\gamma_{m}\right)} t^{(k)} \gamma_{k}\left(s^{(l)}\right) V_{k, l}^{m, \alpha} \psi_{\alpha}^{(m)},
\end{aligned}
$$

which is (2.6).

For the tensor product of the intertwiners, let $s \in \operatorname{Hom}\left(\alpha_{\lambda_{1}}, \alpha_{\mu_{1}}\right), t \in$ $\operatorname{Hom}\left(\alpha_{\lambda_{2}}, \alpha_{\mu_{2}}\right)$. Then, $s \alpha_{\lambda_{1}}(t) \in \operatorname{Hom}\left(\alpha_{\lambda_{1}} \cdot \alpha_{\lambda_{2}}, \alpha_{\mu_{1}} \cdot \alpha_{\mu_{2}}\right)$. We compute $s \alpha_{\lambda_{1}}(t)$ in the case $s=s^{(k)} \psi^{(k)}$ and $t=t^{(l)} \psi^{(l)}$.

$$
\begin{aligned}
s \alpha_{\lambda_{1}}(t) & =s^{(k)} \psi^{(k)} t^{(l)} \varepsilon\left(\lambda_{1}, \gamma_{l}\right)^{*} \psi(l) \\
& =s^{(k)} \gamma_{k}\left(t^{(l)}\right) \gamma_{k}\left(\varepsilon\left(\gamma_{\lambda_{1}}, \gamma_{l}\right)^{*}\right) \psi^{(k)} \psi^{(l)} \\
& =\sum_{\gamma_{m} \in \Delta_{0}^{d}} \sum_{\alpha=1}^{d\left(\gamma_{m}\right)} s^{(k)} \gamma_{k}\left(t^{(l)}\right) \gamma_{k}\left(\varepsilon\left(\lambda_{1}, \gamma_{l}\right)^{*}\right) V_{k, l}^{m, \alpha} \psi_{\alpha}^{(m)}
\end{aligned}
$$

which is (2.7).

For the $*$-operation of the intertwiners, let $t=\sum_{\sigma, i} t_{i}^{(\sigma)} \psi_{i}^{(\sigma)}$. We check the condition for $t=t^{(\sigma)} \psi^{(\sigma)}$.

$$
\left(t^{(\sigma)} \psi^{(\sigma)}\right)^{*}=\psi^{(\sigma)^{*}} t^{(\sigma)^{*}}=R_{\sigma}^{*} \psi^{(\bar{\sigma})} t^{(\sigma)^{*}}=R_{\sigma}^{*} \bar{\sigma}\left(t^{(\sigma)^{*}}\right) \psi^{(\bar{\sigma})},
$$

which is (2.8).

Thus, the image of the $\alpha$-induction is the crossed product $\Delta \rtimes_{0} \Delta^{d}$ in the sense of Müger.

The last claim is immediate from the definitions of $\left(\Delta \cap \Delta^{d^{\prime}}\right) \rtimes \Delta^{d}$ and $\left(\Delta \cap \Delta^{d^{\prime}}\right)^{\alpha}$.

\section{Longo-Rehren inclusions $A \supset B_{\Delta} \supset B_{\hat{\Delta}}$}

Let $\Delta$ be a subset of $\operatorname{End}(M)_{0}$ with a finite braided system $\Delta_{0}, \hat{\Delta} \supset \Delta$ its non-degenerate extension. The following definition was first introduced by Ocneanu [26].

Definition 4.1. The non-degenerate extension $\hat{\Delta} \supset \Delta$ is called minimal if $\hat{\Delta} \cap \Delta^{\prime}=\Delta^{d}$.

Remark that we have $\operatorname{dim} \hat{\Delta}=\operatorname{dim} \Delta \operatorname{dim} \Delta^{d}$ if the extension is minimal.

We assume the minimality of the non-degenerate extension $\hat{\Delta} \supset \Delta$ in the sequel.

Let $\left\{T\left(\begin{array}{l}\zeta \\ \xi, \eta\end{array}\right)\right\}_{i=1}^{N_{\xi, \eta}^{\zeta}}$ be an orthonormal basis of $\operatorname{Hom}(\zeta, \xi \cdot \eta), \xi, \eta, \zeta \in \Delta_{0}$. Let $M$ be the opposite algebra of $M$ and $j: M \longrightarrow M^{o p}$ the anti-linear 
isomorphism. We set $A=M \otimes M^{o p}, \xi^{o p}=j \cdot \xi \cdot j$, and $\hat{\xi}=\xi \otimes \xi^{o p}$. For the isometries $\left\{V_{\xi}\right\}_{\xi \in \Delta_{0}} \subset A$ satisfying $\sum_{\xi \in \Delta_{0}} V_{\xi} V_{\xi}^{*}=1$, we define

$$
\gamma_{\Delta}(x)=\sum_{\xi \in \Delta_{0}} V_{\xi} \hat{\xi}(x) V_{\xi}^{*}
$$

Let $V_{\Delta} \in \operatorname{Hom}(i d, \gamma), W_{\Delta} \in \operatorname{Hom}\left(\gamma, \gamma^{2}\right)$ be isometries defined by

$$
\begin{aligned}
& V_{\Delta}=V_{i d_{M}}, \\
& W_{\Delta}=\sum_{\xi, \eta, \zeta \in \Delta_{0}} \sqrt{\frac{d(\xi) d(\eta)}{\operatorname{dim} \Delta d(\zeta)}} V_{\xi} \hat{\xi}\left(V_{\eta}\right) T_{\xi, \eta}^{\zeta} V_{\zeta}^{*},
\end{aligned}
$$

where $T_{\xi, \eta}^{\zeta}=\sum_{i=1}^{N_{\xi, \eta}^{\zeta}} T\left(\begin{array}{l}\zeta \\ \xi, \eta\end{array}\right)_{i} \otimes j\left(T\left(\begin{array}{l}\zeta, \eta \\ \xi\end{array}\right)\right.$ $)$. Then, one can construct a subfactor $B_{\Delta}$ of $A$ such that $\gamma_{\Delta}: A \longrightarrow B_{\Delta}$ is the canonical endomorphism of the inclusion $A \supset B_{\Delta}$ [18. We call the inclusion $A \supset B_{\Delta}$ the Longo-Rehren inclusion.

In a similar manner, we can construct the Longo-Rehren inclusion $A \supset$ $B_{\hat{\Delta}}$. By their constructions, we have the inclusions $A \supset B_{\Delta} \supset B_{\hat{\Delta}}$.

We define $D(\Delta)$ to be the set of endomorphisms $\rho \in \operatorname{End}\left(B_{\Delta}\right)_{0}$ such that $\left[\iota_{\Delta}\right][\rho]$ is a finite direct sum of sectors in the decompositions of $\{[\xi \otimes$ $\left.\left.i d^{o p}\right]\left[\iota_{\Delta}\right]\right\}_{\xi \in \Delta_{0}}$, where $\iota_{\Delta}$ is the inclusion map $\iota_{\Delta}: B_{\Delta} \hookrightarrow A$. We call $D(\Delta)$ the quantum double of $\Delta$. (For a categorical interpretation of the quantum double, see [23].) In Corollary $7.2[13$, it is proved that $D(\hat{\Delta})$ is equivalent to $\hat{\Delta} \otimes \hat{\Delta}^{o p}$ as modular categories.

Proposition 4.2. We assume that $\Delta^{d} \cong U(G)$, where $G$ is an abelian group. Then, there exists an outer action $\alpha$ of $G$ on $B_{\hat{\Delta}}$ and the subfactor $B_{\Delta} \supset B_{\hat{\Delta}}$ is isomorphic to $B_{\hat{\Delta}} \rtimes_{\alpha} G \supset B_{\hat{\Delta}}$.

Proof. Let $\iota_{1}: B_{\hat{\Delta}} \hookrightarrow B_{\Delta}$ be the inclusion map. Then, by Theorem 7.4 in [13, we have $\left[\bar{\iota}_{1} \iota_{1}\right]=\oplus_{\xi \in \Delta_{0}^{\prime}}\left[{\widehat{\rho_{\xi, \bar{\xi}}}}^{+-}\right]$. By the minimality of the nondegenerate extension, this is $\left[\bar{\iota}_{1} \iota_{1}\right]=\bigoplus_{\xi \in \Delta_{0}^{d}}\left[{\widehat{\rho_{\xi, \bar{\xi}}}}^{+-}\right]$. Since $G \cong \hat{G}$ as groups and $d\left({\widehat{\rho_{\xi, \bar{\xi}}}}^{+-}\right)=d\left(\rho_{\xi}\right)=1$ for each $\xi \in \hat{\Delta}_{0},{\widehat{\rho_{\xi, \bar{\xi}}}}^{+-}$is an automorphism labeled by $G$. Then, by Theorem 4.1 in [12], there exists an outer action $\alpha$ of $G$ on $B_{\hat{\Delta}}$ and the dual inclusion of $B_{\Delta} \supset B_{\hat{\Delta}}$ is $B_{\Delta} \supset B_{\Delta}{ }^{G}$. Hence, $B_{\Delta} \supset B_{\hat{\Delta}}$ is isomorphic to $B_{\hat{\Delta}} \rtimes_{\alpha} G \supset B_{\hat{\Delta}}$.

Theorem 4.3. Let $D(\Delta)$ be the quantum double of $\Delta$. Then, under the assumptions in Proposition 4.2. $D(\Delta)=\left(\hat{\Delta} \otimes \hat{\Delta}^{o p} \cap \Delta^{d^{\prime}}\right) \rtimes \Delta^{d}$, where the embedding $\iota_{\Delta^{d}}: \Delta^{d} \hookrightarrow \hat{\Delta} \otimes \widehat{\Delta}^{o p}$ is given by $\iota_{\Delta^{d}}(\sigma)=\left(\sigma, \sigma^{o p}\right)$.

Proof. First, we may assume that $D(\hat{\Delta})=\hat{\Delta} \otimes \hat{\Delta}^{o p}$ by thanks to Corollary 7.2 in [13. By its construction, $M \rtimes_{\alpha} G$ can be viewed as $M \rtimes \hat{G}$. Then, we may apply Proposition 4.2 to $\hat{\Delta} \otimes \hat{\Delta}^{o p} \cap \Delta^{d^{\prime}}$ to get the crossed product $\left(\hat{\Delta} \otimes \hat{\Delta}^{o p} \cap \Delta^{d^{\prime}}\right) \rtimes \Delta^{d}$ in $\operatorname{End}\left(B_{\Delta}\right)_{0}$. 
By Lemma 7.6 in [13], the image of the $\alpha$-induction in Proposition 3.9] is in $D(\Delta)$. Thus, $\left(\hat{\Delta} \otimes \hat{\Delta}^{o p} \cap \Delta^{d^{\prime}}\right) \rtimes \Delta^{d}$ is a full subcategory of $D(\Delta)$.

We compute the dimension of $\left(\hat{\Delta} \otimes \hat{\Delta}^{o p} \cap \Delta^{d^{\prime}}\right) \rtimes \Delta^{d}$.

$$
\begin{aligned}
\operatorname{dim}\left(\hat{\Delta} \otimes \hat{\Delta}^{o p} \cap \Delta^{d^{\prime}}\right) \rtimes \Delta^{d} & =\frac{\operatorname{dim} \hat{\Delta} \otimes \hat{\Delta}^{o p} \cap \Delta^{d^{\prime}}}{\operatorname{dim} \Delta^{d}}=\frac{\operatorname{dim} \hat{\Delta} \otimes \hat{\Delta}^{o p}}{\left(\operatorname{dim} \Delta^{d}\right)^{2}} \\
& =\left(\frac{\operatorname{dim} \hat{\Delta}}{\operatorname{dim} \Delta^{d}}\right)^{2}=(\operatorname{dim} \Delta)^{2} \\
& =(\operatorname{dim} D(\Delta))^{2},
\end{aligned}
$$

where we used the minimality of the extension $\hat{\Delta} \supset \Delta$ for the fourth equality.

Thus, $\operatorname{dim} D(\Delta)=\operatorname{dim}\left(\hat{\Delta} \otimes \hat{\Delta}^{o p} \cap \Delta^{d^{\prime}}\right) \rtimes \Delta^{d}$, and this implies $D(\Delta)=$ $\left(\hat{\Delta} \otimes \hat{\Delta}^{o p} \cap \Delta^{d^{\prime}}\right) \rtimes \Delta^{d}$.

\section{Application to the Reshetikhin-Turaev in- variants for 3-manifolds}

We apply Theorem 4.3 to the Reshetikhin-Turaev invariant of 3-manifolds constructed from the quantum double $D(\Delta)$ to get a simpler description of it in this case. Before we state Theorem, we collect some general results on a premodular category.

Lemma 5.1. Let $\mathcal{M}$ be a premodular category, $\mathcal{P}$ the non-degenerate extension of $\mathcal{M}$ and $\mathcal{D}$ be degenerates of $\mathcal{M}$, i.e., $\mathcal{D}=\mathcal{M} \cap \mathcal{M}^{\prime}$. Then, we have

$$
\sum_{\omega \in \mathcal{M}_{0}} N_{\eta \bar{\zeta}}^{\omega} d(\omega)=d(\eta \bar{\zeta}) \chi_{\mathcal{M}}(\eta \bar{\zeta})
$$

where $\chi_{\mathcal{M}}(\xi)=1$ if $\xi \in \mathcal{M}, 0$ otherwise.

Proof. We compute $\sum_{\xi \in \mathcal{D}_{0}} S^{\prime}(\xi, \eta) S^{\prime}(\xi, \bar{\zeta})$ in different ways.

On one hand, we have

$$
\begin{aligned}
\sum_{\xi \in \mathcal{D}_{0}} S^{\prime}(\xi, \eta) S^{\prime}(\xi, \bar{\zeta}) & =\sum_{\xi \in \mathcal{D}_{0}} \sum_{\omega \in \mathcal{P}_{0}} d(\xi) N_{\eta \bar{\zeta}}^{\omega} S^{\prime}(\xi, \omega) \\
& =\sum_{\omega \in \mathcal{P}_{0}} N_{\eta \bar{\zeta}}^{\omega} \sum_{\xi \in \mathcal{D}_{0}} d(\xi) S^{\prime}(\xi, \omega) \\
& =\sum_{\omega \in\left(\mathcal{P} \cap \mathcal{D}^{\prime}\right)_{0}} N_{\eta \bar{\zeta}}^{\omega} d(\omega) \operatorname{dim} \mathcal{D}
\end{aligned}
$$

where we used $\sum_{\xi \in \mathcal{D}_{0}} d(\xi) S^{\prime}(\xi, \omega)=d(\omega) \operatorname{dim} \mathcal{D} \chi_{\mathcal{P} \cap \mathcal{D}^{\prime}}(\omega)$ in Lemma 2.13 in [25] for the third equality.

On the other hand,

$$
\begin{aligned}
\sum_{\xi \in \mathcal{D}_{0}} S^{\prime}(\xi, \eta) S^{\prime}(\xi, \bar{\eta}) & =\sum_{\xi \in \mathcal{D}_{0}} S^{\prime}(\xi, \eta \bar{\zeta}) d(\xi) \\
& =d(\eta \bar{\zeta}) \operatorname{dim} \mathcal{D} \chi_{\mathcal{P} \cap \mathcal{D}^{\prime}}(\eta \bar{\zeta})
\end{aligned}
$$


Thus, $\sum_{\omega \in\left(\mathcal{P} \cap \mathcal{D}^{\prime}\right)_{0}} N_{\eta \bar{\zeta}}^{\omega} d(\omega)=d(\eta \bar{\zeta}) \chi_{\mathcal{P} \cap \mathcal{D}^{\prime}}(\eta \bar{\zeta})$ with $\mathcal{P} \cap \mathcal{D}^{\prime}=\mathcal{M}$ implies the claim.

Let $\mathcal{C}$ be a premodular category. Let $L$ be a framed link with $n$ components in the 3 -sphere. We denote the invariant of the colored framed link by $F_{\mathcal{C}}(L, \lambda)$, where $\lambda=\left(\lambda_{1}, \cdots, \lambda_{n}\right) \in \mathcal{C}_{0}^{n}$. Set

$$
\{L\}_{\mathcal{C}}=\sum_{\lambda \in \mathcal{C}_{0}^{n}} \prod_{i=1}^{n} d\left(\lambda_{i}\right) F_{\mathcal{C}}(L ; \lambda) .
$$

We may assume that a closed 3-manifold $M$ is obtained from surgery along the framed link $L$ in the 3 -sphere $S^{3}$. Namely, $M=\partial W_{L}$, where $W_{L}$ is the 4-manifold obtained by gluing $n$ 2-handles to the 4-ball $B^{4}$ along $L \subset S^{3}=$ $\partial B^{4}$. We denote the signature of $W_{L}$ by $\sigma(L)$.

Let $\mathcal{C}$ be a modular category and we set $\Delta_{\mathcal{C}}=\sum_{\xi \in \mathcal{C}_{0}} t_{\xi}^{-1} d(\xi)^{2}$ and $D_{\mathcal{C}}=$ $(\operatorname{dim} \mathcal{C})^{1 / 2}$. The Reshetikhin-Turaev invariant $\tau_{\mathcal{C}}$ is defined by

$$
\tau_{\mathcal{C}}(M)=\left(\Delta_{\mathcal{C}}\right)^{\sigma(L)} D_{\mathcal{C}}^{-\sigma(L)-n-1}\{L\}_{\mathcal{C}}
$$

See 31] for the details of the definition.

Lemma 5.2. Let $\mathcal{C}$ be a premodular category with $\mathcal{C} \cap \mathcal{C}^{\prime}=\mathcal{D}$ and $L$ be a framed link with $n$ components. Then, we have

$$
\{L\}_{\mathcal{C}}=(\operatorname{dim} \mathcal{D})^{n}\{L\}_{\mathcal{C} \rtimes \mathcal{D}}
$$

Proof. This is immediate from Remarques 2.1 1) and Proposition 3.7 1) in [5].

We now go back in the case of braided $C^{*}$-tensor categories $\hat{\Delta}$ and $\Delta$ associated with subfactors. Recall that we have assumed the minimality of the non-degenerate extension $\hat{\Delta} \supset \Delta$. For $\lambda, \mu \in \hat{\Delta}$, we put

$$
[\lambda, \mu]_{\Delta}=\frac{1}{\operatorname{dim} \hat{\Delta}} \sum_{\nu \in \Delta_{0}} N_{\lambda \bar{\mu}}^{\nu} d(\nu)
$$

Theorem 5.3. Let $M$ be a closed 3-manifold obtained from surgery along the framed link $L$ with $n$ components. Then, the Reshetikhin-Turaev invariant for $D(\Delta)$ is given by

$$
\tau_{D(\Delta)}(M)=\frac{1}{\operatorname{dim} \Delta} \sum_{\lambda, \mu \in \hat{\Delta}_{0}^{n}} \prod_{i=1}^{n}\left[\lambda_{i}, \mu_{i}\right]_{\Delta} F_{\hat{\Delta}}(L ; \lambda) \overline{F_{\hat{\Delta}}(L ; \mu)} .
$$

Proof. Since $\Delta_{D(\Delta)}=D_{D(\Delta)}$, we have

$$
\tau_{D(\Delta)}(M)=\frac{1}{(\operatorname{dim} \Delta)^{n+1}} \sum_{\tilde{\xi} \in D(\Delta)_{0}^{n}} \prod_{i=1}^{n} d\left(\tilde{\xi}_{i}\right) F_{D(\Delta)}(L ; \tilde{\xi})
$$


Then, by Theorem 4.3 and Lemma 5.2

$$
\tau_{D(\Delta)}(M)=\frac{1}{(\operatorname{dim} \Delta)^{n+1}\left(\operatorname{dim} \Delta^{d}\right)^{n}} \sum_{\tilde{\zeta} \in\left(\hat{\Delta} \otimes \hat{\Delta}^{o p} \cap \Delta^{d^{\prime}}\right)_{0}^{n}} \prod_{i=1}^{n} d\left(\tilde{\zeta}_{i}\right) F_{D(\Delta)}(L ; \tilde{\zeta}) .
$$

We note that for $\tilde{\zeta} \in\left(\Delta \otimes \Delta^{o p} \cap \Delta^{d^{\prime}}\right)_{0}$ there exist $\lambda, \mu \in \hat{\Delta}_{0}$ such that $\tilde{\zeta}=\lambda \otimes$ $\mu^{o p}$. With this and Lemma 5.1. we have $d(\tilde{\zeta}) \chi_{\hat{\Delta} \cap \Delta^{d^{\prime}}}(\lambda \bar{\mu})=d(\lambda) d(\bar{\mu}) \chi_{\hat{\Delta} \cap \Delta^{d^{\prime}}}(\lambda \bar{\mu})=$ $\operatorname{dim} \hat{\Delta}[\lambda, \mu]_{\Delta}$

Hence, the right hand side of (5.19) is

$\frac{(\operatorname{dim} \hat{\Delta})^{n}}{(\operatorname{dim} \Delta)^{n+1}\left(\operatorname{dim} \Delta^{d}\right)^{n}} \sum_{\lambda, \mu \in \hat{\Delta}_{0}^{n}} \prod_{i=1}^{n}\left[\lambda_{i}, \mu_{i}\right]_{\Delta} F_{\hat{\Delta} \otimes \hat{\Delta}^{o p}}\left(L ; \lambda_{1} \otimes \mu_{1}^{o p}, \lambda_{2} \otimes \mu_{2}^{o p}, \cdots, \lambda_{n} \otimes \mu_{n}^{o p}\right)$.

Since we have the equality $F_{\hat{\Delta} \otimes \hat{\Delta}^{o p}}\left(L ; \lambda_{1} \otimes \mu_{1}^{o p}, \lambda_{2} \otimes \mu_{2}^{o p}, \cdots, \lambda_{n} \otimes \mu_{n}^{o p}\right)=$ $F_{\hat{\Delta}}\left(L ; \lambda_{1}, \cdots, \lambda_{n}\right) \overline{F_{\hat{\Delta}}\left(L ; \mu_{1}, \cdots, \mu_{n}\right)}$ and the minimality of the non-degenerate extension $\hat{\Delta} \supset \Delta$, we have

$$
\tau_{D(\Delta)}(M)=\frac{1}{\operatorname{dim} \Delta} \sum_{\lambda, \mu \in \hat{\Delta}_{0}^{n}} \prod_{i=1}^{n}\left[\lambda_{i}, \mu_{i}\right]_{\Delta} F_{\hat{\Delta}}(L ; \lambda) \overline{F_{\hat{\Delta}}(L ; \mu)} .
$$

Remark 5.4. With Theorem 5.2 in [16], which claims that the Turaev-ViroOcneanu invariant for $\Delta$ is equal to the Reshetikhin-Turaev invariant for $D(\Delta)$, Theorem 5.3 proves a slightly different statement of Theorem 3.2 in [26] in the special case, although Ocneanu claims that it also holds true for $G$, a non-abelian group.

\section{References}

[1] M. F. Atiyah, Topological quantum field theories, Publ. Math. I.H.E.S. 68 (1989) 175-186.

[2] J. Böckenhauer, D. E. Evans, Modular invariants, graphs, and $\alpha-$ induction for nets of subfactors. I, Commun. Math. Phys. 197 (1998), 361-386.

[3] J. Böckenhauer, D. E. Evans, Modular invariants, graphs, and $\alpha$ induction for nets of subfactors. II, Commun. Math. Phys. 200 (1999), 57-103.

[4] J. Böckenhauer, D. E. Evans, Modular invariants, graphs, and $\alpha$ induction for nets of subfactors. III, Commun. Math. Phys. 205 (1999), 183-228. 
[5] A. Bruguières, Catégories prémodulaires, modularisations et invariants de variété de dimension 3, Math. Ann. 316 (2000), 215-236.

[6] S. Doplicher, J.E. Roberts, Endomorphisms of $C^{*}$-algebras, crossed products and duality for compact groups, Ann. Math. 130 (1989), 75119 .

[7] S. Doplicher, J.E. Roberts, A new duality theory for compact groups, Invent. Math. 98 (1989) 157-218.

[8] D. E. Evans, Y. Kawahigashi, Orbifold subfactors from Hecke algebras, Commun. Math. Phys. 165 (1994) 445-484.

[9] D. E. Evans, Y. Kawahigashi, On Ocneanu's theory of asymptotic inclusions for subfactors, topological quantum field theories and quantum doubles, Internat. J. Math. 6 (1995) 205-228.

[10] D. E. Evans, Y. Kawahigashi, Orbifold subfactors from Hecke algebras II, Commun. Math. Phys. 196 (1998) 331-361.

[11] S. Goto, Orbifold construction for non-AFD subfactors, Internat. J. Math. 5 (1994), 725-746.

[12] M. Izumi, Applications of fusion rules to classification of subfactors, Publ. RIMS, 27, (1991), 953-994.

[13] M. Izumi, The structures of sectors associated with the Longo-Rehren inclusions I. General theory, Commun. Math.Phys. 213 (2000) 127-179.

[14] C. Kassel, Quantum groups, Springer-Verlag 1995.

[15] Y. Kawahigashi, On flatness of Ocneanu's connections on the Dynkin diagrams and classification of subfactors, J. Funct. Anal. 127 (1995), 63-107.

[16] Y. Kawahigashi, N. Sato, M. Wakui, (2+1)-dimensional topological quantum field theory from subfactors and Dehn surgery formula for 3-manifold invariants, preprint, math.OA/0208238, 2002.

[17] T. Kohno, T. Takata, Symmetry of Witten's 3-manifold invariants for $\operatorname{sl}(n, \mathbb{C})$, J. Knot Theory and its Ramif. 2 (1993), 149-169.

[18] R. Longo, K.-H. Rehren, Nets of subfactors, Rev. in Math. Phys. 7 (1995), 567-597.

[19] R. Longo, J. E. Roberts, A theory of dimension, K-theory 11 (1997), 103-159.

[20] T. Masuda, An analogue of Longo's canonical endomorphism for bimodule theory and its application to asymptotic inclusions, Internat. J. Math. 8 (1997), 249-265. 
[21] T. Masuda, Extension of automorphisms of a subfactor to the symmetric enveloping algebra, Internat. J. Math. 12 (2001), 637-659.

[22] M. Müger, On charged fields with group symmetry and degeneracies of Verlinde's matrix S, Ann. Inst. Henri Poincaré, 71 (1999), 359-394.

[23] M. Müger, From subfactors to categories and topology II. The quantum double of tensor categories and subfactors, J. Pure Appl. Alg. 180 (2003), 159-219.

[24] M. Müger, Galois theory for braided tensor categories and the modular closure, Adv. in Math. 150 (2000), 151-201.

[25] M. Müger, On the structure of modular categories, Proc. London Math. Soc. 87 (2003), 291-308.

[26] A. Ocneanu, Chirality for operator algebras, 39-63, in "Subfactors", ed. by H. Araki, et al., World Scientific, 1994.

[27] K.-H. Rehren, Braid group statistics and their super selection rules, in "The Algebraic Theory of Superselection sectors", ed. by D. Kastler, World Scientific, 1990.

[28] K.-H. Rehren, Markov traces as characters for local algebras, Nucl. Phys. B(Proc. Suppl.) 18B (1990), 259-268.

[29] J. E. Roberts, Crossed products of von Neumann algebras by group duals, Sympos. Math. XX (1976), 335-363.

[30] S. F. Sawin, Jones-Witten invariants for nonsimply connected Lie groups and the geometry of the Weyl alcove, Adv. in Math. 165 (2002), $1-34$.

[31] V. G. Turaev, Quantum invariants of knots and 3-manifolds, Walter de Gruyter 1994.

[32] H. Wenzl, Hecke algebras of type $A_{n}$ and subfactors, Invent. Math. 92 (1988), 345-383.

[33] F. Xu, New braided endomorphisms from conformal inclusions, Commun. Math. Phys. 192 (1998) 349-403.

[34] S. Yamagami, Group symmetry in tensor categories and duality for orbifolds, J. Pure Appl. Alg. 167 (2002), 83-128. 